\title{
Null-Steering Reflectarrays for 5G Backhaul Networks Limited by Interference
}

\author{
Xavier Artiga \\ ${ }^{1}$ Centre Tecnològic de Telecomunicacions de Catalunya (CTTC), Barcelona, Spain, xavier.artiga@cttc.es
}

\begin{abstract}
Future 5G backhaul networks in dense small cell deployments will require high gain antennas with beam- and null-steering capabilities. In this framework this paper analyzes the null-steering capabilities of reflectarray antennas, as a low cost alternative to traditional antenna arrays. In particular, a random search algorithm is used to show that null-steering can be performed even with 1-bit reflectarrays, but that the synthesized null-depth and width is much more sensitive to aperture phase errors than the main beam gain. Multi-feed reflectarrays are then proposed to overcome this sensitivity but at the expense of higher system complexity and cost.
\end{abstract}

Index Terms-reflectarray, null-steering, phase error, phase quantization, $5 \mathrm{G}$, backhaul networks.

\section{INTRODUCTION}

Next $5 \mathrm{G}$ networks will relay in dense deployments of small cells operating at millimeter wave frequencies in order to cope with the unprecedented traffic increase forecasted for the coming years. Such dense networks impose several requirements to the antennas used in their backhaul segment. In particular, high gain is required to overcome the large pathloss experienced at mm-wave region; steerable solutions are needed to allow changes in the physical network topology for a flexible operation [1]; and null-steering capabilities are required to mitigate interferences between nodes which could become a limiting factor due to the cell densification.

Reflectarrays (RA) are a suitable antenna candidate to fulfill all the above requirements. Indeed, they are a promising low cost alternative to traditional massive arrays, since their spatial feeding mechanism avoids the need of bulky and complex beamforming networks. Although a lot of work has been done in the study of reconfigurable RAs over the past years [2], their null-steering capabilities remain as an unexplored area. Null-steering has been addressed in the context of traditional arrays [3], but specific RA characteristics such as the spherical illumination or the use of quantization down to 1-bit needs further research. Interestingly, 1-bit RA configurations are affected by a gain reduction around $4 \mathrm{~dB}$ and a rise of their side-lobe levels [4], what reinforces the need of null-steering.

In this paper a random search algorithm for performing null-steering is proposed and used to evaluate the impact of quantization and phase errors on the null quality. In view of the observed extreme sensitivity of the null-depth to phase errors in the aperture, multi-feed RA configurations are explored next. In this case, the RA is used only to steer the main beam whereas the null is synthesized by the multiple feeds. This scheme is similar to array fed parabolic reflectors [5], but with the advantage that the RA aperture still can steer the beam in a significant angular range.

The random search algorithm for null synthesis is detailed in Section II, whereas Sections III and IV evaluate single- and multi-feed RA configurations. Finally Section IV present the paper conclusions.

\section{NULL SINTHESYS WITH RANDOM SEARCH}

This paper addresses the scenario in which the given side-lobe levels of a beam steering RA are not low enough to mitigate an interference coming from a closely located and/or high power transmitter. Therefore a pattern synthesis algorithm that aims to minimize the gain at a specific interfered angle (or angular sector) while not affecting the main beam in excess, is proposed. The selected solution is based on an iterative random search [6], because of its simplicity and capability of ease including the quantization constraints in the optimization process. Fig. 1 depicts a flow chart of this algorithm.

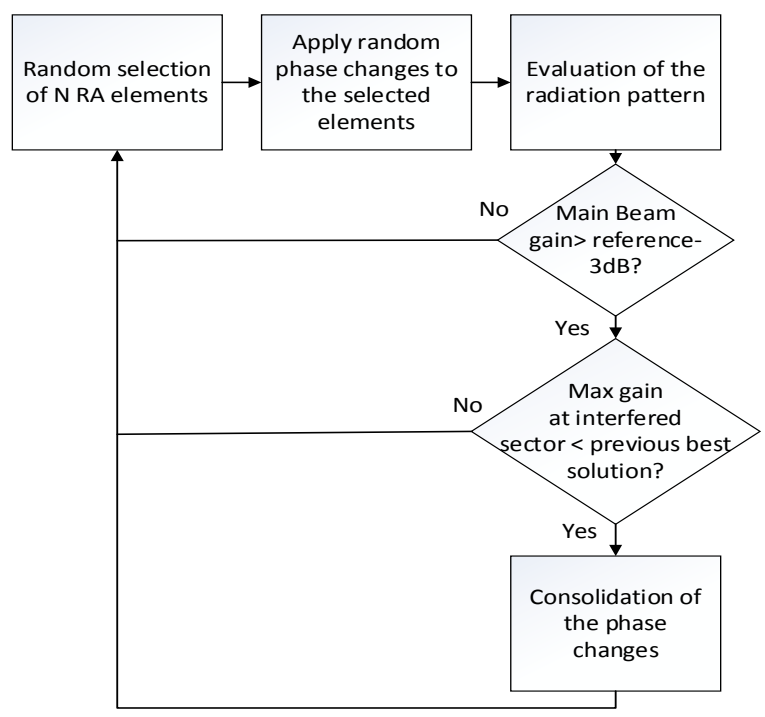

Fig. 1. Flow chart of the random search iteration.

Basically in a first step $N$ RA elements are randomly selected and their phases are randomly changed. The value of $N$ and the amount of phase change can be varied in order to control the speed of convergence and the effects over the main beam (larger phase changes would have a larger impact 
on it). The radiation pattern of the resulting phase distribution is then evaluated, and this last is only consolidated in the case that the gain at the interfered locations is lower than the previous and the constraint on the main beam gain reduction is fulfilled. Without the loss of generality, here it is assumed that the interfered location is characterized by an angular spread of $1^{\circ}$, thus the minimization of the RA gain in $1^{\circ}$ sectors is pursued. In addition, the acceptable gain reduction is fixed to $3 \mathrm{~dB}$ with respect to simple beam steering RA pointing at the same location.

\section{SINGLE FEED NULL-STEERING}

The analysis of the capabilities of a single feed RA considers a centre-fed circular RA with 631 elements with inter-element spacing of half-wavelength; $F / D=1$, amplitude tapering at the RA edge around $-10 \mathrm{~dB}$, and ideal cells (reflection amplitude $=1$ ). The radiation patterns are calculated using the aperture-field method [7].

First, the straightforward method sometimes used in pure beam steering applications, based on optimizing and analog phase distribution and then quantizing it, is evaluated. As exemplified in Fig. 2, the null depth is largely affected when applying aggressive quantization. Even, the performance with 5-bits is significantly worse than using full phase resolution. In contrast, the main beam gain only suffers the well predicted gain reduction, which indicates that the beamand null-steering mechanisms are totally different. It is worse remarking here that digital RAs usually employ one or two bits since the biasing complexity becomes unaffordable when the number of bits increases.

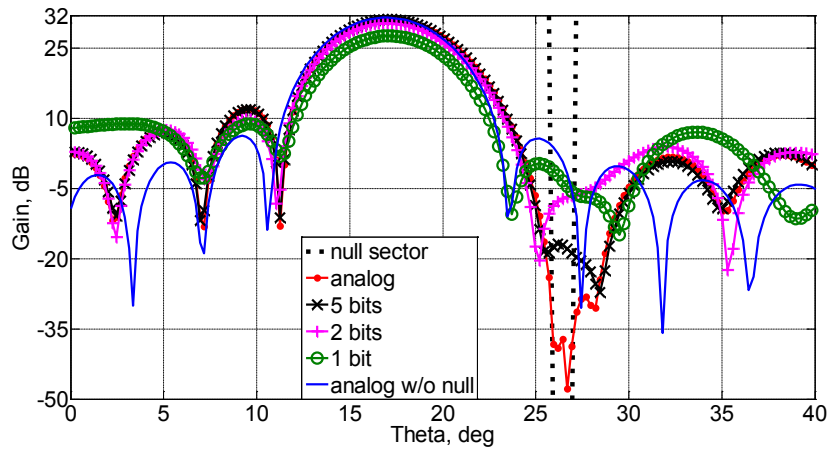

Fig. 2. Radiation patterns at the $\varphi=0$ plane with main beam at $(\theta, \varphi)=\left(17^{\circ}\right.$, $\left.0^{\circ}\right)$ and $1^{\circ}$ interfering sector at $(\theta, \varphi)=\left(26.5^{\circ}, 0^{\circ}\right)$ when an anlog phase distribution is optimized and then quantized.

Now the alternative of directly including the quantization constraints in the optimization method is explored. In other words, the random phase changes applied to the randomly selected RA elements, are forced to be one of the possible phase states. Fig. 3 demonstrates that this method mostly preserves the null-depths obtained by the full resolution scheme even with 1-bit quantization. The main beam gains in this case are a little bit lower than those of Fig. 2 since the optimization algorithm allowed maximum reduction of $3 \mathrm{~dB}$.

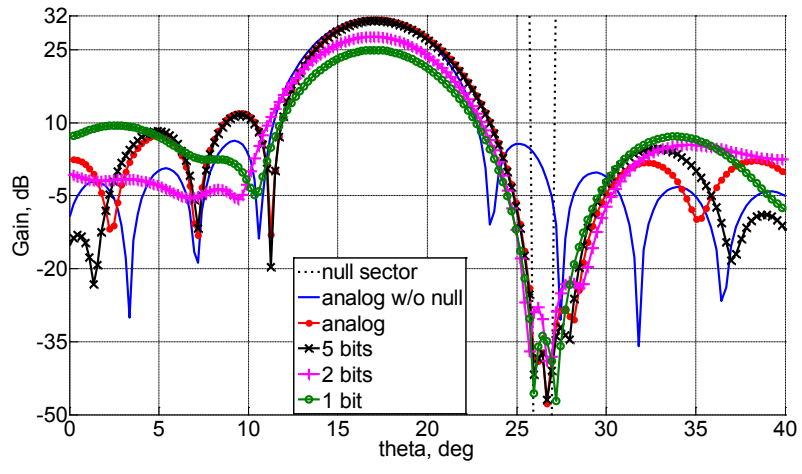

Fig. 3. Radiation patterns at the $\varphi=0$ plane with main beam at $(\theta, \varphi)=\left(17^{\circ}\right.$, $\left.0^{\circ}\right)$ and $1^{\circ}$ interfering sector at $(\theta, \varphi)=\left(26.5^{\circ}, 0^{\circ}\right)$ when the quantized phase distribution is directly optimized.

In order to stress the different mechanisms of beam and null-steering next it is evaluated the impact of small phase errors on top of an optimized 1-bit phase distribution. It is well-known that the main beam is not affected by small random errors since the quantization errors for 1-bit RAs can be as large as $90^{\circ}$. However, the null-depth can be significantly affected as shown next. For this evaluation Montecarlo simulations with 150 realizations are performed. In each realization the main beam and the null directions are randomly selected in the range of $-180^{\circ}<\varphi \leq 180^{\circ}$ and $0 \leq \theta \leq 30^{\circ}$. Overlapping between the main beam and the null sector is avoided. In addition, for each main beam/null sector position, 100 realizations adding different randomly generated phase errors are considered, which results in a total of 1500 realizations. The random phase errors are characterized by a Gaussian variable with $0^{\circ}$ mean and standards deviation of $1^{\circ}, 3^{\circ}, 5^{\circ}$ and $8^{\circ}$. Fig. 4 shows the simulation results in terms of the null depth degradation with respect to the null depth obtained with the optimized 1-bit phase distribution without randomly generated errors.

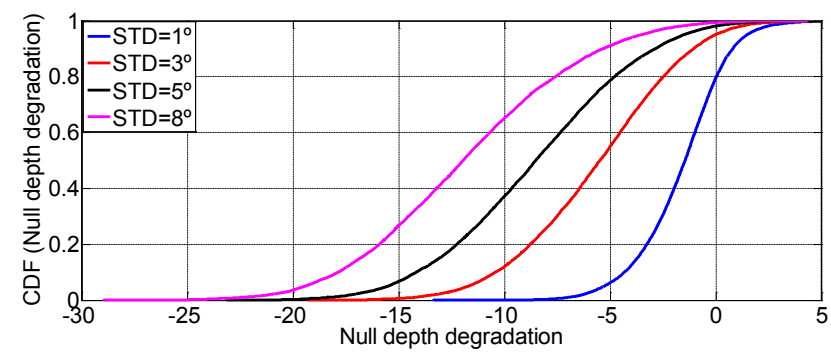

Fig. 4. CDF of the null depth degradation with respect to the optimized 1bit phase distribution.

A null-depth degradation greater than $10 \mathrm{~dB}$ is observed in the $12 \%, 37 \%$, and $65 \%$ of the cases when random phase errors with standard deviations of $3^{\circ}, 5^{\circ}$ and $8^{\circ}$ are added, respectively. Note that in a real RA implementation there could be many sources of small phase errors such as: implementation errors, incidence angles not taken in account in the phase evaluation, periodic boundary assumptions, etc. Moreover the sensitivity of the null-depth to small phase has also a significant impact on the null-steering bandwidth. 
Indeed the null-steering bandwidth will be lower than the beam-steering one since this last is more robust to the phase errors induced by the frequency change.

\section{MULTI-FEED NULL-STEERING}

This section proposes a multi-feed RA configuration in order to overcome the sensitivity to small phase errors of single feed configurations. In this architecture, the RA only performs beam steering whereas the signals at each of the feeds are combined in order to synthesize the required null. The concept is similar to array fed parabolic reflectors, but the use of a reconfigurable RA as the reflector provides large steerable ranges.

Fig. 5-6 shows the resilience of this scheme to phase errors in the RA aperture. Here, three feeds are located at $(x, y)=[(-\lambda, 0),(0,0),(\lambda, 0)]$. A Linearly constrained minimum variance (LCMV) beamformer forcing $-60 \mathrm{~dB}$ nulls with respect to the main beam gain is used. It can be observed that the synthesized null remains constant even adding random phase errors with Gaussian distributions of $0^{\circ}$ mean and standard deviations as high as $90^{\circ}$. Interestingly, in this last case the main beam is slightly affected while the null depth remains the same. The radiation pattern of a center fed single feed RA is shown as a reference.

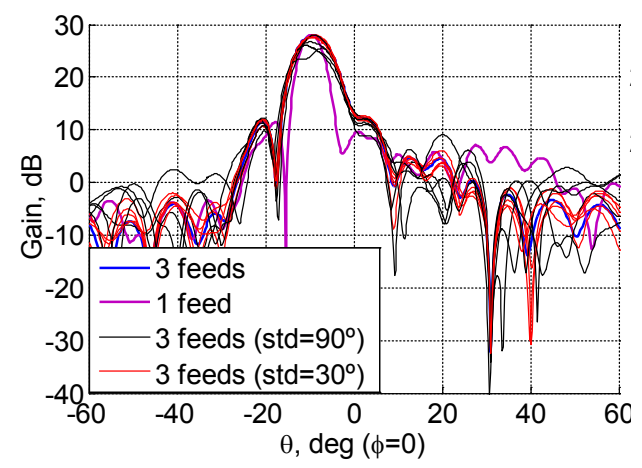

Fig. 5. Radiation patterns at the $\varphi=0$ plane with main beam at $(\theta, \varphi)=\left(-10^{\circ}\right.$, $\left.0^{\circ}\right)$ and an interferer at $(\theta, \varphi)=\left(31^{\circ}, 0^{\circ}\right)$.

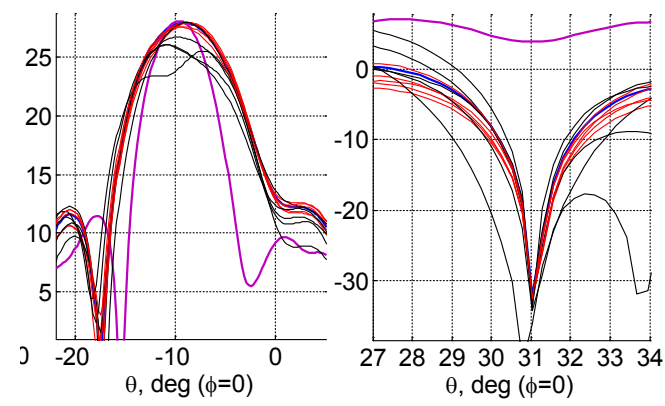

Fig. 6. Left: zoom at the main beam. Right: zoom at the synthesized null.

The other benefits of this scheme are provided by the use of digital beamforming. On the one side, it enables the use standard closed form beamfomers such as the LCMV, thus avoiding the need of complex iterative optimization processes. On the other side, the digital processing may allow extending the null-bandwidth through the use of filter bank processing techniques. However, the multi-feed scheme presents also important limitations. First, the number of nulls that can be synthesized is $\mathrm{N}-1$, being $\mathrm{N}$ the number of feeds. Second, the nulls are synthesized in a single direction, thus several nulls (or derivative constraints) must be co-located in order nullify sectors of a few degrees. It must be noted that mm-wave backhaul links above roof tops have been characterized with angular spreads up to $5^{\circ}$, caused by reflections on nearby objects [8]. Fig. 7 shows an example of two co-located nulls. The same 3 -feed system as in Fig. 5 is considered. It can be observed that the two collocated nulls consume degrees of freedom, and as a result, the main beam is slightly affected. Therefore, the cost and complexity of the multi-feed solution grows as the number of nulls to synthesize and their widths increase.

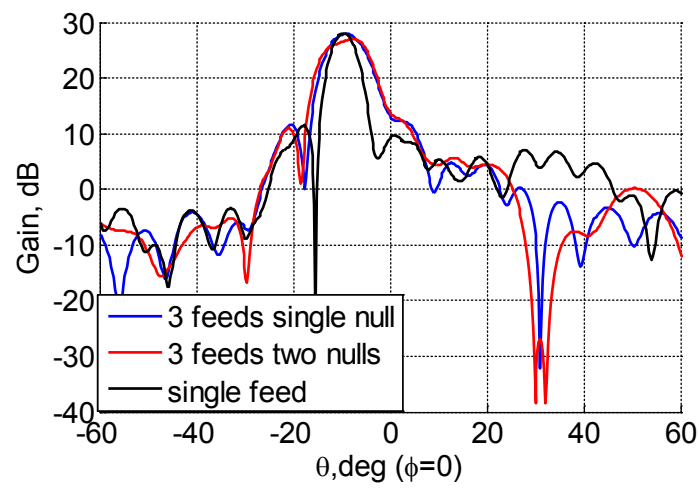

Fig. 5. Radiation patterns at the $\varphi=0$ plane with main beam at $(\theta, \varphi)=\left(-10^{\circ}\right.$, $\left.0^{\circ}\right)$ and an interfer at at $(\theta, \varphi)=\left(31^{\circ}, 0^{\circ}\right)$.

Finally, the study of the multi-feed RA revealed that in some cases, the array response (amplitude and phase of the radiation pattern) in the desired and interfered directions are similar, what does not allow synthesizing a deep null without greatly affecting the main beam. This depends on the directions of the nulls and the main beam, as well as on the position and number of feeds, as shown in Fig. 6. A joint optimization of the RA phase distribution and the beamformer may help mitigating this issue but remains as an unexplored area of research.

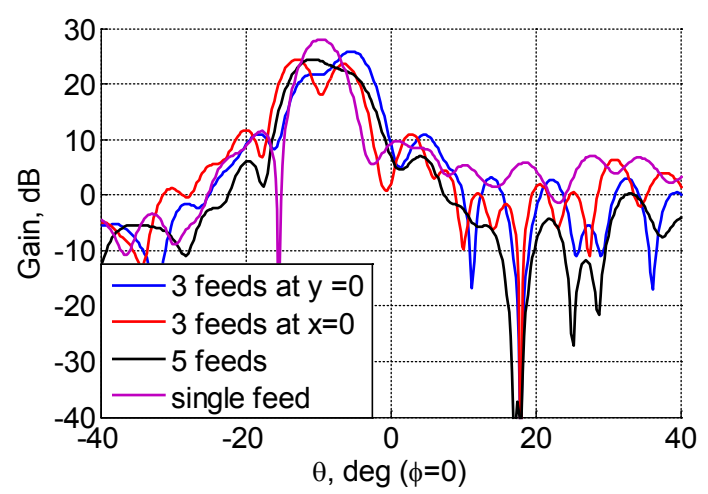

Fig. 5. Radiation patterns at the $\varphi=0$ plane with main beam at $(\theta, \varphi)=\left(-10^{\circ}\right.$, $\left.0^{\circ}\right)$ and an interfer at at $(\theta, \varphi)=\left(18^{\circ}, 0^{\circ}\right)$. 


\section{CONCLUSIONS}

This paper studied the null-steering capabilities of single and multi-feed RA antennas. In the case of single feed RAs, the analysis revealed that the beam- and null-steering mechanisms present a different behavior with respect to quantization and phase errors. In particular, null-steering is very sensitive to phase errors which have two main implications: (i) null-synthesis algorithms must directly include the quantization constraints in the optimization process; and (ii) all sources of phase errors must be perfectly characterized in order to synthesize the nulls. As an alternative, adaptive null steering control may also allow preserving the theoretical null depths. Resilience to phase errors, can be achieved by a multi feed configuration with digital beamforming. However, this scheme increases the system complexity and cost and is limited by the total number of feeds used. Moreover, further work is needed to jointly optimize the number and position of the feeds, the RA phase distribution and the beamformer design in order to preserve both null-depths and main beam gains.

\section{ACKNOWLEDGMENT}

This work received funding from the European Union's Horizon 2020 research and innovation programme under grant agreement No 645047 (SANSA); the Spanish Ministry of Economy and Competitiveness (Ministerio de Economia y Competitividad) under project TEC2014-59255-C3-1-R; and from the Catalan Government (2014SGR1567).

[8] A. Maltsev and et al, "D5.1 - Channel Modeling and Characterization," MiWEBA Project (FP7-ICT-608637), Public Deliverable, Jan. 2014.

\section{REFERENCES}

[1] X. Artiga, J. Núñez-Martínez, A. Pérez-Neira, G. Lendrino, J. Faré, G. Ziaragkas, "Terrestrial-Satellite Integration in Dynamic 5G Backhaul Networks" 2016 8th Advanced Satellite Multimedia Systems Conference and the 14th Signal Processing for Space Communications Workshop (ASMS/SPSC), Palma de Mallorca, Spain.

[2] S. V. Hum and J. Perruisseau-Carrier, "Reconfigurable Reflectarrays and Array Lenses for Dynamic Antenna Beam Control: A Review," in IEEE Transactions on Antennas and Propagation, vol. 62, no. 1, pp. 183-198, Jan. 2014.

[3] M. Caillet, Y. M. M. Antar and M. Clénet, "Amplitude and phase quantization effects on synthesized array factors including one beam and one prescribed null," Phased Array Systems and Technology (ARRAY), 2010 IEEE International Symposium on, Waltham, MA, 2010, pp. 974-981J.

[4] S. Ebadi, R. V. Gatti and R. Sorrentino, "Linear reflectarray antenna design using 1-bit digital phase shifters," 2009 3rd European Conference on Antennas and Propagation, Berlin, 2009, pp. 37293732.

[5] S. K. Sharma, S. Chatzinotas, J. Grotz and B. Ottersten, "3D Beamforming for Spectral Coexistence of Satellite and Terrestrial Networks," Vehicular Technology Conference (VTC Fall), 2015 IEEE 82nd, Boston, MA, 2015, pp. 1-5.

[6] B. Widrow and J. McCool, "A comparison of adaptive algorithms based on the methods of steepest descent and random search," in IEEE Transactions on Antennas and Propagation, vol. 24, no. 5, pp. 615-637, Sep 1976.

[7] P. Nayeri, A. Z. Elsherbeni and F. Yang, "Radiation Analysis Approaches for Reflectarray Antennas [Antenna Designer's Notebook]," in IEEE Antennas and Propagation Magazine, vol. 55, no. 1, pp. 127-134, Feb. 2013. 